\title{
Exploring the Masses of Exotic Heavy Pentaquarks
}

\section{Ballari Chakrabarti*Department of physics, Jogamaya Devi College, Kolkata, India.}

E-mail: ballari_chakrabarti@yahoo.co.in

The masses of crypto exotic heavy pentaquarks have been probed considering a di-hadronic state consisting of a meson and a baryon. The interaction between the hadrons is assumed to be Van der Waals' type of molecular interaction and we have estimated the binding energies of the states. A spin interaction has also been considered. Masses of the crypto heavy pentaquarks such as $P_{s}^{*}(1), P_{s}^{*}(2), P_{b}^{*}(1), P_{b}^{*}(2)$, have been predicted . Moreover we have also calculated the masses of exotic pentaquarks for the charm and the bottom families constituting a heavy quark.

38th International Conference on High Energy Physics 3-10 August 2016

Chicago, USA

${ }^{*}$ Speaker. 


\section{Introduction}

The existence of pentaquark charmonium states with the decay of $\Lambda_{b}^{0}\left(\Lambda_{b}^{0} \rightarrow J / \Psi K^{-} p\right)$ has been reported by LHCb [1] recently. The intermediate states have been identified as $P_{c}^{*}(4380)$ and $P_{c}^{*}(4450)$. New impetus has been put forward to the study of the properties and dynamics of the multiquark states by Jaffe et al [2]. The works of Lipkin [3], Gignoux et al [4], Monemzadeh et al [5], Diakonov [6] in the context of the exotic doublets $(c u u d \bar{s})(c u d d \bar{s})$, Bicudo [7]for possible crypto heptaquark hadrons with exotic flavors, Kopeliovich et al [8] for cryptoexotic pentaquarks with hidden beauty are noteworthy.

\section{Methodology}

In the present work exotic heavy pentaquarks have been described as di-hadronic molecules consisting of a meson and a baryon held together by Van der Waals' type of molecular interaction. The mass formula for the low-lying di-hadronic molecule is taken as

$$
M_{T o t}=M_{1}+M_{2}+E_{B E}+E_{S D}
$$

where $M_{1}$ and $M_{2}$ represent the masses of the constituent hadrons respectively, $E_{B E}$ represents the binding energy of the di-hadronic system and $E_{S D}$ represents the spin dependent term. The binding energy can be expressed as

$$
E_{B E}=<\Psi\left(r_{12}\right)\left|V\left(r_{12}\right)\right| \Psi\left(r_{12}\right)>
$$

where $r_{12}$ is the radius parameter of the di-hadronic molecule and $V(r)$ is the interaction potential taken as Van der Waals' type [9] which is expressed as

$$
V\left(r_{12}\right)=-\frac{k_{m o l}}{r_{12}} e^{-C^{2} r_{12}^{2} / 2}
$$

where $k_{m o l}$ is the residual strength of the strong interaction molecular coupling and $C$ is the effective color screening of the confined gluons. $\Psi\left(r_{12}\right)$ is the wave function of the dihadronic state and is assumed to be in the context of the statistical model as [10],

$$
\left|\Psi\left(r_{12}\right)\right|^{2}=\frac{315}{64 \pi r_{0}^{9 / 2}}\left(r_{0}-r_{12}\right)^{3 / 2} \theta\left(r_{0}-r_{12}\right)
$$

corresponding to the linear type of background potential. $r_{0}$ is the radius of the di-hadronic molecule, $\theta\left(r_{0}-r_{12}\right)$ is the usual step function. Employing the additive rule for the radii of constituent hadrons i.e. $r_{0}=r_{1}+r_{2}, r_{1}$ and $r_{2}$ representing the individual radii of the hadrons constituting the molecule, we have executed the binding energy using equations (2.2),(2.3) and (2.4), which yields

$$
E_{B E}=\frac{2.25 k_{m o l}}{r_{0}}\left[{ }_{2} F_{2}[(1.5,1),(2.75,2.25),-\beta]\right]
$$

if $R e \beta>0{ }_{2} F_{2}$ is the relevant hypergeometric function and $\beta=C^{2} r_{12}^{2} / 2$. Input values are $C=$ $50 \mathrm{MeV}$ [11] $k_{\text {mol }}=0.65$ [10]. The radii considered are $r(p)=6 \mathrm{GeV}^{-1}, r(n)=4.7 \mathrm{GeV}^{-1}, r(K)=$ $4.77 \mathrm{GeV}^{-1}, r(\Phi)=5.0 \mathrm{GeV}^{-1}, r(\Upsilon)=1.63 \mathrm{GeV}^{-1}, r\left(D^{-}\right)=4.97 \mathrm{GeV}^{-1}, r\left(B_{s}\right)=3.67 \mathrm{GeV}^{-1}$, $r\left(D_{s}\right)=4.8 \mathrm{GeV}^{-1}, r\left(B^{o}\right)=3.82 \mathrm{GeV}^{-1}, r(\Sigma)=3.9 \mathrm{GeV}^{-1}, r(\Delta)=5.977 \mathrm{GeV}^{-1}$. 


\begin{tabular}{|c|c|c|c|c|}
\hline Particles & Approach & Mass $(\mathrm{GeV})$ & Expt. Mass (GeV) & Others Mass (GeV) \\
\hline$P_{s}^{*}(1 / 2)^{+}$ & $p+\Phi$ & 2.110 & - & $2.303[8]$ \\
\hline $\begin{array}{l}P_{s}^{*}(3 / 2)^{+} \\
(u u d s \bar{s})\end{array}$ & $\Delta+\Phi$ & 2.381 & - & $2.373[8]$ \\
\hline$P_{b}^{*}(1 / 2)^{+}$ & $p+\Upsilon$ & 10.639 & - & $10.743[8]$ \\
\hline $\begin{array}{l}P_{b}^{*}(3 / 2)^{+} \\
(u u d b \bar{b})\end{array}$ & $\Delta+\Upsilon$ & 10.881 & - & $10.813[8]$ \\
\hline $\begin{array}{l}\Theta_{c}^{0} \\
(u u d d \bar{c})\end{array}$ & $\begin{array}{l}p+D^{-} \\
n+\bar{D}^{0}\end{array}$ & $\begin{array}{l}2.656 \\
2.670\end{array}$ & $\begin{array}{l}3.099 \pm 0.003 \pm 0.005 \\
{[13]}\end{array}$ & $\begin{array}{l}2.650[6] \\
2.710[3]\end{array}$ \\
\hline $\begin{array}{l}N_{c}^{0} \\
(u u d s \bar{c})\end{array}$ & $p+D^{0}$ & 2.752 & - & $2.870[3]$ \\
\hline $\begin{array}{l}\Xi_{c}^{0} \\
(u u s s \bar{c})\end{array}$ & $\Sigma^{+}+D^{0}$ & 2.905 & - & $3.135[3]$ \\
\hline $\begin{array}{l}\Theta_{c s}^{++} \\
(u u d c \bar{s})\end{array}$ & $p+\overline{D^{0}}$ & 2.751 & - & $2.427[5]$ \\
\hline $\begin{array}{l}\Theta_{b}^{+} \\
(u u d d \bar{b})\end{array}$ & $\begin{array}{l}p+B^{0} \\
n+B^{+}\end{array}$ & $\begin{array}{l}6.043 \\
6.067\end{array}$ & - & $6.050[3]$ \\
\hline $\begin{array}{l}N_{b}^{+} \\
(u u d s \bar{b})\end{array}$ & $p+B_{s}$ & 6.127 & - & $6.210[3]$ \\
\hline $\begin{array}{l}\Xi_{b}^{+} \\
(\text {uuss } \bar{b})\end{array}$ & $\Sigma^{+}+B_{s}$ & 6.366 & - & $6.351[3]$ \\
\hline $\begin{array}{l}\Theta_{b s}^{+} \\
(u u d b \bar{s})\end{array}$ & $p+\bar{B}_{s}$ & 6.128 & - & $5.752[5]$ \\
\hline
\end{tabular}

Table 1: Masses of cryptoexotic heavy pentaquarks

The spin hyperfine interaction can be expressed as,

$$
E_{S D}=\frac{8 \alpha_{s}}{9 M_{1} M_{2}} \mathbf{S}_{\mathbf{1}} . \mathbf{S}_{\mathbf{2}}|\Psi(0)|^{2}
$$

$\alpha_{s}$ is the strong interaction constant, $\mathbf{S}_{\mathbf{1}}$ and $\mathbf{S}_{\mathbf{2}}$ are the spins of the hadrons involved. $|\Psi(0)|^{2}$ is the di-hadronic wave function at the origin. With $\alpha_{s}=0.59$ for light hadrons and 0.2 for heavier sector [12] $E_{S D}$ has been estimated subsequently. $P_{s}^{*}(1 / 2)^{+}$and $P_{s}^{*}(3 / 2)^{+}$have been assumed to have configuration as proton- $\Phi$ state and $\Delta-\Phi$ state and $P_{b}^{*}(1 / 2)^{+}$and $P_{b}^{*}(3 / 2)^{+}$as proton- $\Upsilon$ state and $\Delta-\Upsilon$ state respectively. Based on this formulation several cryptoexotic heavy pentaquark masses such as $\Theta_{c}^{0}, \Theta_{b}^{+}, \Theta_{c s}^{++}, \Theta_{b s}^{+}, N_{c}^{0}, N_{b}^{+}, \Xi_{0}^{0}, \Xi_{b}^{+}$have also been calculated and have been displayed in the Table I along with the comparison with others.

\section{Conclusion}

We have investigated several cryptoexotic pentaquark systems as hadronic composites of a meson and a baryon. Our results are in good agreement with the other theoretical works as well as experiments wherever available. Experimental mass of $\Theta_{c}^{0}$ [13] is available but that is not confirmed 
later [14]. We hope that this ambiguity could be understood in near future experiments. In this context it may be pertinent to state that in our previous work we have already estimated the masses of recently reported $P_{c}^{*}(4380)$ and $P_{c}^{*}(4450)$ as $4168 \mathrm{MeV}$ and $4491 \mathrm{MeV}$ describing these as $p+J / \Psi$ and $\Delta+J / \Psi$ di-hadronic states respectively [15]. We want to explore the properties of other exotic pentaquark families. Although the production rates are probably very low, these can be looked for at LHC, Fermilab, B-factories, RHIC etc. The predictions made in this work may inspire the future experiments.

\section{Acknowledgement}

Author is thankful to University Grants Commission, New Delhi for financial assistance.

\section{References}

[1] R. Aaij et al. (LHCb Collab.), Observations of $J / \Psi p$ resonances consistent with pentaquark states in $\Lambda_{b}^{0} \rightarrow J / \Psi K^{-}$p, Phys. Rev. Lett. 115 (2015) 072001.

[2] R. L. Jaffe et al., Diquarks and exotic spectroscopy, Phys. Rev. Lett. 91(2003) 232003; M. Karlinar et al.,A diquark-triquark model for the KN pentaquark, Phys. Lett.B 575 (2003) 249.

[3] H. J. Lipkin, New possibilities for exotic hadrons anticharmed baryons, Phys. Lett. $B 195$ (1987) 484.

[4] C. Gignoux et al., Possibility of stable multiquark baryons, Phys. Lett. B 193 (1987) 323.

[5] M. Monemzadeh et al., Calculating masses of pentaquarks composed of baryons and mesons, [hep-ph/1601.00642].

[6] D. Diakonov, Prediction of new charmed and bottom exotic pentaquarks, [hep-ph/1003.2157].

[7] P. Bicudo, Exotic pentaquarks, crypto-heptaquarks and linear three hadronic molecules, [hep-ph/0410097].

[8] V. Kopeliovich et al., Simple estimates of the masses of pentaquarks with hidden beauty or strangeness, [hep-ph/1510.05958].

[9] P. C. Vinodkumar et al., Effect of the confined gluons in quark-quark interaction, Pramana J. Phys. 39 (1992) 47.

[10] B. Chakrabarti et al., On some properties of di-hadronic states, Phys. Scr. 79 (2009) 025103.

[11] A. K. Rai et al., Low-lying di-hadronic states in relativistic harmonic model, Ind. J. Phys. 80 (2006) 387.

[12] W. Lucha et al., Bound states of quarks, Phys. Rep. 200 (1991) 168.

[13] A. Aktas et al. (H1 Collab.), Evidence for a narrow anti-charmed baryon state Phys. Lett.B 588 (2004) 17.

[14] J. M. Link et al. (FOCUS Collab.) Search for a strongly decaying neutral charmed pentaquark Phys. Lett. B 622 (2005) 229.

[15] R. Ghosh et al., The masses of $P_{c}^{*}(4380)$ and $P_{c}^{*}(4450)$ as di-hadronic states, J. Mod. Phys. 6 (2015) 2070. 